\title{
Addition of multiple limiting resources reduces grassland diversity
}

\author{
W. Stanley Harpole ${ }^{1,2,3}$, Lauren L. Sullivan ${ }^{4}$, Eric M. Lind ${ }^{4}$, Jennifer Firn ${ }^{5}$, Peter B. Adler ${ }^{6}$, Elizabeth T. Borer ${ }^{4}$, Jonathan Chase ${ }^{2,3}$, \\ Philip A. Fay ${ }^{7}$, Yann Hautier ${ }^{8}$, Helmut Hillebrand ${ }^{9}$, Andrew S. MacDougall ${ }^{10}$, Eric W. Seabloom ${ }^{4}$, Ryan Williams ${ }^{11}$, \\ Jonathan D. Bakker ${ }^{12}$, Marc W. Cadotte ${ }^{13}$, Enrique J. Chaneton ${ }^{14}$, Chengjin Chu ${ }^{15}$, Elsa E. Cleland ${ }^{16}$, Carla D’Antonio ${ }^{17}$, \\ Kendi F. Davies ${ }^{18}$, Daniel S. Gruner ${ }^{19}$, Nicole Hagenah ${ }^{20}$, Kevin Kirkman²0, Johannes M. H. Knopss ${ }^{21}$, Kimberly J. La Pierre ${ }^{22}$, \\ Rebecca L. McCulley ${ }^{23}$, Joslin L. Moore ${ }^{24}$, John W. Morgan ${ }^{25}$, Suzanne M. Prober ${ }^{26}$, Anita C. Risch ${ }^{27}$, Martin Schuetz ${ }^{27}$, \\ Carly J. Stevens ${ }^{28}$ \& Peter D. Wragg ${ }^{29}$
}

\begin{abstract}
Niche dimensionality provides a general theoretical explanation for biodiversity - more niches, defined by more limiting factors, allow for more ways that species can coexist ${ }^{1}$. Because plant species compete for the same set of limiting resources, theory predicts that addition of a limiting resource eliminates potential trade-offs, reducing the number of species that can coexist ${ }^{2}$. Multiple nutrient limitation of plant production is common and therefore fertilization may reduce diversity by reducing the number or dimensionality of belowground limiting factors. At the same time, nutrient addition, by increasing biomass, should ultimately shift competition from belowground nutrients towards a one-dimensional competitive trade-off for light ${ }^{3}$. Here we show that plant species diversity decreased when a greater number of limiting nutrients were added across $\mathbf{4 5}$ grassland sites from a multi-continent experimental network ${ }^{4}$. The number of added nutrients predicted diversity loss, even after controlling for effects of plant biomass, and even where biomass production was not nutrient-limited. We found that elevated resource supply reduced niche dimensionality and diversity and increased both productivity ${ }^{5}$ and compositional turnover. Our results point to the importance of understanding dimensionality in ecological systems that are undergoing diversity loss in response to multiple global change factors.
\end{abstract}

The search for the mechanisms underlying the coexistence of multiple species was inspired by Darwin's observations of the problem of the 'entangled bank', or how different checks on the growth of individuals underlie the number of species found together ${ }^{6}$. One of the most general theoretical explanations for this problem is that greater dimensionality, or number of non-overlapping ecological niches, allows for the coexistence of a greater number of species ${ }^{1,7}$. However, plant coexistence challenges this understanding: rather than occupying unique resource niches, plants share and are limited by the same essential resources ${ }^{8}$. The coexistence of plants competing for the same resources therefore requires stoichiometric and physiological trade-off differences for shared limiting resources ${ }^{2}$. Furthermore, plant resources are spatially separated, with elemental nutrients (for example, nitrogen, phosphorus, potassium) and water acquired belowground and light aboveground. This suggests that two, non-independent resourcebased mechanisms could maintain plant diversity: multi-dimensional trade-offs for belowground limiting nutrients, juxtaposed with a onedimensional trade-off for light aboveground.

Resource competition theory predicts that addition of a limiting resource makes that resource non-limiting, thereby eliminating a competitive trade-off contributing to coexistence ${ }^{2}$. Because some factor must ultimately limit growth, resource additions will lead to a reduction in the number and a shift in the identity of growth-limiting factors. In the case of plants, addition of multiple nutrients should reduce the dimensionality of belowground resource trade-offs, increase biomass production, and ultimately shift the prevailing form of resource competition towards a single, aboveground limiting resource, light ${ }^{3,5}$. Support for this hypothesis has been demonstrated in four grassland experiments. All of these experiments found plant biomass production was limited by multiple resources, and diversity decreased as a function of the number of belowground resources made non-limiting ${ }^{5,9-11}$. These results are consistent with the hypothesis that multi-dimensional tradeoffs for belowground resources, and light competition mediated by aboveground biomass production, might jointly contribute to maintaining plant diversity in natural communities. Although multiple limitation of primary producer communities is common ${ }^{12}$, a recent global study demonstrated substantial site-level variation in the number and identity of co-limiting resources, with around $25 \%$ of sites showing no evidence that biomass production was nutrient limited ${ }^{13}$. The question remains whether the dimensionality of nutrient resources might contribute to plant diversity independently of the presumed importance of indirect effects of biomass on diversity.

Here we tested for loss of species diversity in response to multiple nutrient addition $s^{5}$ using the Nutrient Network, a globally-distributed,

\footnotetext{
${ }^{1}$ Department of Physiological Diversity, Helmholtz Center for Environmental Research - UFZ, Permoserstrasse 15, Leipzig 04318, Germany. ${ }^{2}$ German Centre for Integrative Biodiversity Research

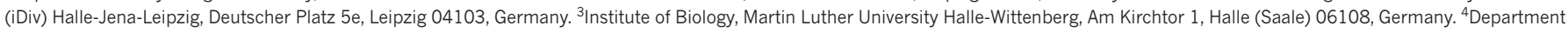
of Ecology, Evolution, and Behavior, University of Minnesota, St Paul, Minnesota 55108, USA. ${ }^{5}$ School of Earth, Environment and Biological Sciences, Queensland University of Technology,

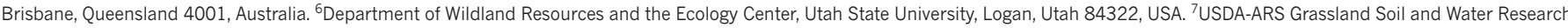

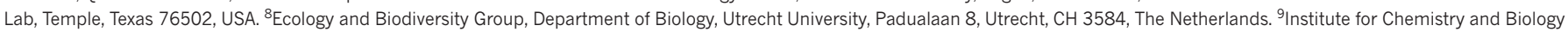

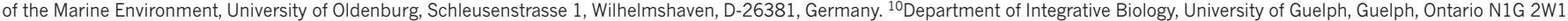

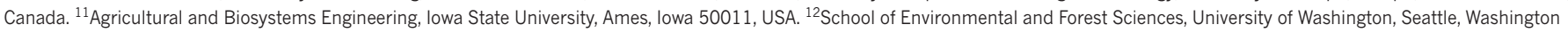
98195, USA. ${ }^{13}$ Department of Biological Sciences, University of Toronto - Scarborough, 1265 Military trail, Toronto, Ontario M1C 1A4, Canada. ${ }^{14}$ IFEVA/CONICET - Departamento de Recursos Naturales y Ambiente, Facultad de Agronomía, Universidad de Buenos Aires. Av. San Martín 4453 (C1417DSE) Buenos Aires, Argentina. ${ }^{15}$ SYSU-Alberta Joint Lab for Biodiversity Conservation, State Key Laboratory of Biocontrol and School of Life Sciences, Sun Yat-sen University, Guangzhou 510275, China. ${ }^{16}$ Ecology, Behavior \& Evolution Section, University of California, La Jolla, San Diego, California 92093, USA. ${ }^{17}$ Department of Ecology, Evolution and Marine Biology, University of California, Santa Barbara, California 93106-9620 USA. ${ }^{18}$ Department of Ecology and Evolutionary Biology, University of Colorado, Boulder, Colorado 80309, USA. ${ }^{19}$ Department of Entomology, University of Maryland, College Park, Maryland 20742, USA. ${ }^{20}$ School of Life Sciences, University of KwaZulu-Natal, Pietermaritzburg 3209, South Africa. ${ }^{21}$ School of Biological Sciences, University of Nebraska, Lincoln, Nebraska 68588 , USA. ${ }^{22}$ Department of Integrative Biology, University of California, Berkeley, California 94720, USA. ${ }^{23}$ Department of Plant and Soil Sciences, University of Kentucky, Lexington, Kentucky 40546, USA. ${ }^{24}$ School of Biological Sciences,

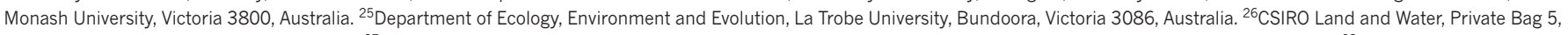
Wembley, Western Australia 6913, Australia. ${ }^{27}$ Swiss Federal Institute for Forest, Snow and Landscape Research, Community Ecology, Birmensdorf 8903 , Switzerland. ${ }^{28}$ Lancaster Environment Centre, Lancaster University, Lancaster LA1 4YQ, UK. ${ }^{29}$ Department of Ecology \& Evolutionary Biology, Yale University, 165 Prospect Street, New Haven, Connecticut 06511, USA.
} 


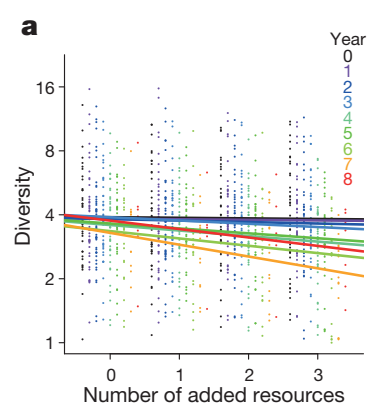

Figure 1 | Biodiversity and number of resources. a, Loss of species diversity with greater number of added resources (effective number of equally abundant species: $\mathrm{ESN}_{\mathrm{PIE}}$ ); this effect increased with years of treatment 1-8 (Extended Data Table 1); year 0 shows pre-treatment diversity. Bold lines show overall mean responses of 45 sites; $y$ axis is log-transformed. b, Greater number of added resources increased

nutrient addition experiment, replicated across grassland sites on six continents (NutNet; http://www.nutnet.org) ${ }^{4}$. We added factorial combinations of phosphorus $(\mathrm{P})$, nitrogen $(\mathrm{N})$, and potassium $\left(\mathrm{K}_{+\mu}\right.$; the $\mathrm{K}$ addition treatment included sulfur and a one-time addition of micronutrients; see Methods), with the aim of removing potential limitations from different combinations of the essential nutrient elements that most strongly affect plant growth in natural and managed systems worldwide ${ }^{13}$. Our treatments varied in the number of elemental resources they contained; hereafter, we use the term 'number of added resources' $(1,2$ or 3$)$ to represent the minimum number of potentially limiting elemental nutrients added (see Methods).

If competition for multiple belowground resources contributes to species coexistence, then diversity should decrease as a function of the number of resources added. Species diversity decreased as more resources were added, and this effect increased with duration of treatment (Fig. 1a and Extended Data Table 1). Greater number of added resources increased the annual rate of diversity loss, even after controlling for differences in experiment duration (Fig. 1b). We found a similar proportional loss of diversity with a greater number of added resources (using the log-ratio effect size of treatment divided by control diversity; Fig. 1b), meaning that in terms of the number of potential species lost, relative diversity losses and annual rate of diversity loss were similar. Sites differed in the size of their species pools, which ranged from 13 to 103 observed species over a three-year period, and we found that the magnitude of diversity loss rate per added resource increased with local species pool size (Fig. 1c).

We found that increasing the number of added resources increased live biomass (Fig. 2a), and decreased the proportion of photosynthetically active radiation (PAR) transmitted through the canopy to the ground surface (Fig. 2b). Further, the amount of litter biomass, which can also contribute to light limitation and diversity $\operatorname{loss}^{14}$ increased with the number of added resources (Fig. 2c). Importantly, despite the complex causal effects of changes in multiple resources on the c

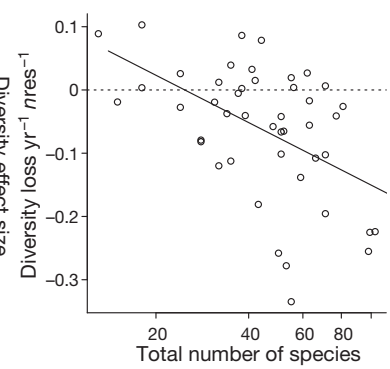

the mean rates of diversity loss per year (filled points; $F_{1,134}=24.8$, $P<0.0001$ ), and the proportional loss of species relative to the controls, shown as the effect size (open points; $F_{1,134}=46.2, P<0.0001$ ). c, Rate of diversity loss per added resource ( $n$ res) was associated with greater total site species number $\left.(\log ), R^{2}=0.25, P=0.0004, n=45\right)$. Error bars show mean $\pm 95 \%$ confidence intervals.

relationship between diversity and biomass, the number of added resources remained a significant predictor of diversity loss, even after controlling for the potential contributing effects of species pool size, live biomass, total cover (a proxy for total plant abundance), light transmittance, and litter mass (Extended Data Tables 2 and 3). If species coexist though trade-offs in resource-ratio requirements, changes in belowground resource supply could cause changes in competitive dominance and lead to species exclusion ${ }^{2}$, independent of aboveground effects of biomass. In a subset of sites that did not show a biomass response to multiple nutrient addition, we nevertheless observed declines in diversity consistent with this theory (Fig. 3a, b: open points, $n=11$ ), similar to sites where biomass production was multiple-resource limited (Fig. 3a, b: filled points, $n=34$ ). Overall, 14 sites of 45 sites in this study showed some type of negative biomass response to N, P or $\mathrm{K}_{+\mu}$ addition suggesting the potential for elevated nutrient concentrations supply to cause negative physiological responses in species not adapted to high nutrient concentrations ${ }^{15}$ or to large stoichiometric imbalances in resource supply ${ }^{16}$.

Diversity loss increased only weakly with biomass increase in plots receiving all three resources, providing some support for indirect effects of biomass as a contributing, but not a sole, mechanism of diversity loss due to fertilisation (Fig. 3c). If species losses were most strongly associated with biomass increases, we would expect the greatest effects on both responses to be associated with the same nutrient addition treatment, but this was true for only 22 of 45 cases (Chi-square, $P<0.0001$ ). The loss of diversity was not driven by the addition of any single added resource (for example, N); greatest diversity loss occurred with the addition of a combination of two or more resources in 31 of 45 cases. These findings further highlight that biomass production and diversity can be controlled differently by multiple resources. Overall, these results support our conclusion that resource niche dimensionality can contribute to species diversity independently of indirect effects mediated by biomass production.

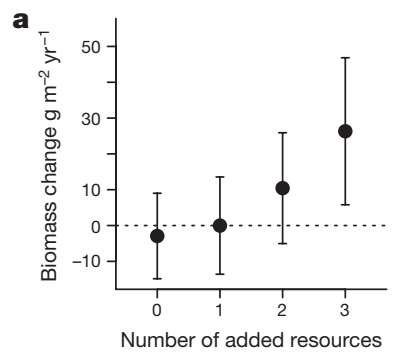

Figure 2 | Biomass and light. a, The rate of live biomass change per year increased with an increasing number of added resources $\left(F_{1,1031}=55.0\right.$, $P<0.0001)$. b, The proportion of photosynthetically active radiation $(\mathrm{PAR})$ reaching the ground surface decreased with a greater number

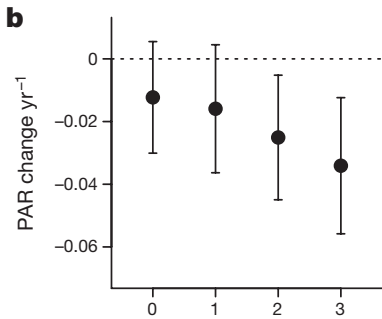

Number of added resources

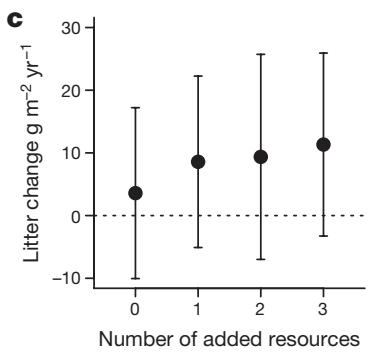

of added resources, expressed as annual rate of change $\left(F_{1,782}=62.4\right.$, $P<0.0001)$. c, The mean rate of litter (dead biomass) change per year increased with the number of added resources $\left(F_{1,783}=4.37, P=0.037\right)$. Error bars show mean $\pm 95 \%$ confidence intervals. 

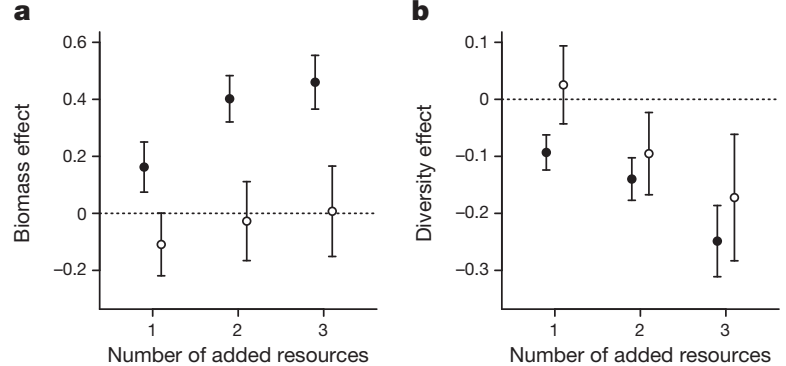

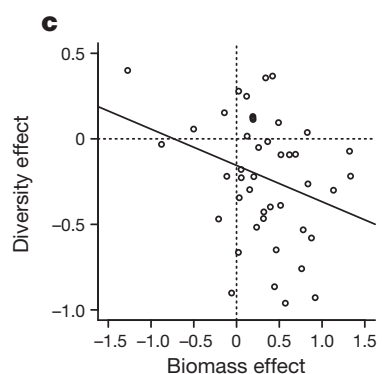

similar diversity loss at sites where biomass production was limited by multiple resources (filled points) and at sites where it was not (open points). c, Negative relationship between the effect of addition of three resources on biomass and diversity (one-tailed test for negative relationship, $\left.R^{2}=0.11, P=0.012, n=45\right)$. Error bars show mean \pm s.e.
For resource dimensionality to contribute to species coexistence, species must trade-off their competitive abilities for different limiting resources, and changes in resource supply ratios should drive species compositional turnover ${ }^{2}$. We found that a greater number of added resources increased the compositional divergence from control plots (Fig. 4a). Plots receiving a single resource treatment (N, P and $\mathrm{K}_{+\mu}$ treatments) diverged as much from each other as they did on average from the control plots (Fig. 4b), consistent with different species trading off competitive abilities for different resources ${ }^{2}$. We found that greater diversity loss was weakly associated with greater community dissimilarity when all three resources were added together (Fig. 4c), suggesting that resource addition caused changes in community composition that were not always associated with diversity loss. Both composition and diversity of communities contribute to ecosystem functioning, and many of the proposed mechanisms of the effect of species diversity on ecosystem function are resource-based ${ }^{17}$. Additionally, nutrient enrichment impacts some groups of species more than others (for example, a loss of native species in favour of exotic grasses ${ }^{18}$ ). Because changes in resource supply led to communities of fewer species and of different compositions, we expect changes in resources, acting through diversity loss, to have both direct and indirect effects on ecosystem functions ${ }^{19}$.

Although our results are consistent with predictions of the resource niche dimension hypothesis, they are also probably conservative. Our experimental design, a factorial manipulation of three resource treatments, represents a lower-bound estimate of the dimensionality of nutrient resources because our $\mathrm{K}_{+\mu}$ treatment included sulfur and up to 10 other macro- and micro-nutrients, of which more than one may have been limiting ${ }^{13}$. Multiple chemical forms of a limiting nutrient can also contribute to species diversity ${ }^{20}$, further expanding potential resource dimensionality. Stronger tests of the role of multiple resource competition for structuring species coexistence require physiological studies quantifying species-specific functional traits and trade-offs ${ }^{21}$, and testing whether species respond to resource treatments similarly in different environments. Deeper mechanistic insight can also be gained by asking how resource-dependent diversity patterns and mechanisms change across scales (for example, from local to regional) in response to global change drivers such as nutrient pollution ${ }^{22}$. Our results point to, but do not distinguish among, the presumed resource competition mechanisms ${ }^{2}$ that underlie the resource dimension hypothesis.

We found that greater diversity loss was associated with soil $\mathrm{P}, \mathrm{K}$, $\mathrm{pH}$ and percentage sand, but not with soil $\mathrm{N}$, or with latitude, or mean annual precipitation (Extended Data Table 4), suggesting that variation in soil properties may influence the degree to which communities respond to changes in resource availability ${ }^{23}$. We did not test or control for other potential limiting factors such as herbivory or water, which can interact with nutrients in complex ways, and themselves contribute to species coexistence. For example, changes in nutrient availability affect photosynthetic tissue quantity and quality, and may alter the pattern and intensity of herbivory ${ }^{24}$, and the level of soil water depletion through transpiration losses. Our multi-year experimental results may still under-estimate nutrient effects when considering that global eutrophication represents a chronic and cumulative environmental change over many decades. Estimating effective upper bounds on ecologically relevant resource dimensionality will depend on the degree to which multiple limiting factors covary, how they change in time and space, and how multiple limiting factors interact with each other in promoting coexistence. Global change is driving environmental conditions beyond multiple planetary boundaries ${ }^{25}$, and changing the limiting factors that structure species diversity ${ }^{26}$. Understanding the mechanisms that underlie diversity loss caused by multiple global change factors is necessary to develop effective management strategies for restoring and preserving Earth's biodiversity.
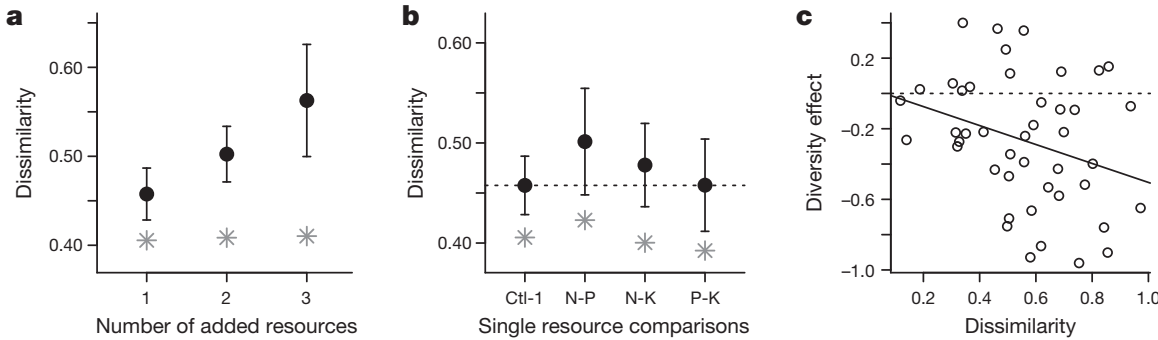

Figure 4 | Community composition. a, Community composition diverged from control plots with greater number of added resources (Bray-Curtis dissimilarity index). Resource addition caused greater dissimilarity of community composition relative to mean pre-treatment dissimilarity, indicated by grey stars. $\mathbf{b}$, Addition of single nutrient additions of N, P or $\mathrm{K}_{+\mu}$ resulted in communities that diverged as much from each other as they did on average from the control plots. Pre-treatment values indicated by grey stars. c, Negative relationship between the effect of addition of three resources on community dissimilarity relative to controls and diversity (one-tailed test for negative relationship, $R^{2}=0.10, P=0.019$, $n=45$ ). Error bars indicate mean $\pm 95 \%$ confidence intervals. 
Online Content Methods, along with any additional Extended Data display items and Source Data, are available in the online version of the paper; references unique to these sections appear only in the online paper.

\section{Received 2 February; accepted 21 July 2016.}

Published online 24 August 2016.

1. Hutchinson, G. E. Concluding remarks. Quant. Biol. 22, 415-427 (1957).

2. Tilman, D. Resource Competition and Community Structure (Princeton Univ. Press, 1982).

3. Hautier, Y., Niklaus, P. A. \& Hector, A. Competition for light causes plant biodiversity loss after eutrophication. Science 324, 636-638 (2009).

4. Borer, E. T. et al. Finding generality in ecology: a model for globally distributed experiments. Methods Ecol. Evol. 5, 65-73 (2014).

5. Harpole, W. S. \& Tilman, D. Grassland species loss resulting from reduced niche dimension. Nature 446, 791-793 (2007).

6. Darwin, C. R. On the Origin of Species (John Murray, 1859).

7. Interlandi, S. J. \& Kilham, S. S. Limiting resources and the regulation of diversity in phytoplankton communities. Ecology 82, 1270-1282 (2001).

8. Hutchinson, G. E. The paradox of the plankton. Am. Nat. 95, 137-147 (1961).

9. Silvertown, J., Biss, P. M. \& Freeland, J. Community genetics: resource addition has opposing effects on genetic and species diversity in a 150-year experiment. Ecol. Lett. 12, 165-170 (2009).

10. Ren, Z. et al. Effects of resource additions on species richness and ANPP in an alpine meadow community. J. Plant Ecol. 3, 25-31 (2010)

11. Harpole, W. S. et al. Nutrient co-limitation of primary producer communities.Ecol. Lett. 14, 852-862 (2011).

12. Elser, J. J. et al. Global analysis of nitrogen and phosphorus limitation of primary producers in freshwater, marine and terrestrial ecosystems. Ecol. Lett. 10, 1135-1142 (2007).

13. Fay, P. A. et al. Grassland productivity limited by multiple nutrients. Nature Plants 1, 15080 (2015)

14. Foster, B. L. \& Gross, K. L. Species richness in a successional grassland: effects of nitrogen enrichment and plant litter. Ecology 79, 2593-2602 (1998).

15. Chapin, F. S. III The mineral nutrition of wild plants. Annu. Rev. Ecol. Syst. 11, 233-260 (1980).

16. Cardinale, B. J., Hillebrand, H., Harpole, W. S., Gross, K. \& Ptacnik, R. Separating the influence of resource 'availability' from resource 'imbalance' on productivity-diversity relationships. Ecol. Lett. 12, 475-487 (2009)

17. Tilman, D., Isbell, F. \& Cowles, J. M. Biodiversity and ecosystem functioning. Annu. Rev. Ecol. Evol. Syst. 45, 471-493 (2014).

18. Seabloom, E. W. et al. Plant species' origin predicts dominance and response to nutrient enrichment and herbivores in global grasslands. Nature Commun. 6, 7710 (2015)

19. Isbell, F. et al. Nutrient enrichment, biodiversity loss, and consequent declines in ecosystem productivity. Proc. Natl Acad. Sci. USA 110, 11911-11916 (2013).
20. von Felten, S. et al. Belowground nitrogen partitioning in experimental grassland plant communities of varying species richness. Ecology $\mathbf{9 0}$, 1389-1399 (2009).

21. Litchman, E. \& Klausmeier, C. A. Trait-based community ecology of phytoplankton. Annu. Rev. Ecol. Evol. Syst. 39, 615-639 (2008).

22. Chase, J. M. \& Knight, T. M. Scale-dependent effect sizes of ecological drivers on biodiversity: why standardised sampling is not enough. Ecol. Lett. 16 (Suppl 1), 17-26 (2013).

23. Flores-Moreno, $\mathrm{H}$. et al. Climate modifies response of non-native and native species richness to nutrient enrichment. Phil. Trans. R. Soc. B 371, 20150273 (2016).

24. Borer, E. T. et al. Herbivores and nutrients control grassland plant diversity via light limitation. Nature 508, 517-520 (2014).

25. Steffen, W. et al. Planetary boundaries: guiding human development on a changing planet. Science 347, 1259855 (2015).

26. Tilman, D. \& Lehman, C. Human-caused environmental change: impacts on plant diversity and evolution. Proc. Natl Acad. Sci. USA 98, 5433-5440 (2001).

Supplementary Information is available in the online version of the paper.

Acknowledgements We thank the Minnesota Supercomputer Institute for hosting project data, the University of Minnesota Institute on the Environment for hosting Nutrient Network meetings, and each site investigator for funding their site-level operations. Network coordination and data management were supported by funds from the National Science Foundation Research Coordination Network (NSF-DEB-1042132) to E.T.B. and E.W.S. from the Long Term Ecological Research program (NSF-DEB-1234162) to the Cedar Creek LTER, and from the Institute on the Environment (DG-0001-13). Konza NutNet site was funded by the Konza Prairie LTER; the Saline Experimental Range NutNet site was funded by a Yale Institute for Biospheric Studies Pilot Grant. Nitrogen fertilizer was donated to the Nutrient Network by Crop Production Services, Loveland, Colorado. We thank N. Gotelli for discussions.

Author Contributions W.S.H. analysed the data and wrote the paper with contributions and input from all authors. L.L.S., E.M.L. and J.F. contributed to data analysis. W.S.H., E.W.S. and E.T.B. developed and framed the research questions. W.S.H., E.W.S., E.T.B. and E.M.L. are Nutrient Network coordinators. All authors collected data used in this analysis. Author contribution matrix provided as Supplementary Table 2.

Author Information Reprints and permissions information is available at www.nature.com/reprints. The authors declare no competing financial interests. Readers are welcome to comment on the online version of the paper. Correspondence and requests for materials should be addressed to W.S.H. (stan.harpole@idiv.de).

Reviewer Information Nature thanks J. Levine, B. Schmid and the other anonymous reviewer(s) for their contribution to the peer review of this work. 


\section{METHODS}

Data reporting. No statistical methods were used to predetermine sample size. The investigators were not blinded to allocation during experiments and outcome assessment.

Experimental design. The Nutrient Network (NutNet) is a collaborative, distributed experimental network ${ }^{4}$. Sites are located across herbaceous terrestrial systems on six continents. Vegetation types represented include grasslands, savannas and meadows and occur across a wide range of climate and environmental factors (Supplementary Table 1). At the 45 sites (on five continents) with appropriate experimental data for these analyses, one year of pre-treatment (year 0 ) data were collected followed by at least 3 years and up to 8 years of treatment data. Individual site experiments share identical design and sampling protocols, with minor site-specific differences in terms of replication and treatment duration (Supplementary Table 1). We applied factorial combinations of nitrogen $(\mathrm{N})$, phosphorus (P), and potassium plus micronutrients, designated here as the $\mathrm{K}_{+\mu}$ treatment, giving eight treatment combinations including the control with no added resources. $\mathrm{N}$ was applied annually at $10 \mathrm{~g} \mathrm{~N} \mathrm{~m}^{-2} \mathrm{yr}^{-1}$ as time-release urea. Ammonium nitrate was used in 2007 at some sites before switching to urea due to restricted availability of ammonium nitrate; we found no differences in the short-term effects of alternative $\mathrm{N}$ sources in a separate experiment at four sites ${ }^{18}$. $\mathrm{P}$ was applied at $10 \mathrm{gP} \mathrm{m}^{-2} \mathrm{yr}^{-1}$ as triple-super phosphate, which also included $\mathrm{Ca}$ at $8.1 \mathrm{~g} \mathrm{Ca} \mathrm{m}^{-2} \mathrm{yr}^{-1}$. The $\mathrm{K}_{+\mu}$ treatment added a mix of $\mathrm{K}$ and $\mathrm{S}\left(10 \mathrm{~g} \mathrm{~K} \mathrm{~m}^{-2} \mathrm{yr}^{-1}\right.$ and $3.9 \mathrm{~g} \mathrm{~S} \mathrm{~m}^{-2} \mathrm{yr}^{-1}$ as potassium sulphate) and micronutrients $\left(100 \mathrm{~g} \mathrm{~m}^{-2} \mathrm{yr}^{-1}\right.$ of a mixture composed of $6 \% \mathrm{Ca}, 3 \% \mathrm{Mg}, 12 \% \mathrm{~S}, 0.1 \% \mathrm{~B}, 1 \% \mathrm{Cu}, 17 \% \mathrm{Fe}, 2.5 \% \mathrm{Mn}$, $0.05 \% \mathrm{Mo}$, and $1 \% \mathrm{Zn}$ ). Micronutrients were only applied during the first treatment year to minimise potential for toxic metal accumulation. Plots were $5 \mathrm{~m} \times 5 \mathrm{~m}$ and randomized within 1 to 6 blocks (Supplementary Table 1), with all eight treatment combinations occurring once per block. Sampling occurred at approximately peak biomass times for each site.

Response measurements. Biodiversity estimates are scale-dependent ${ }^{27}$, and increased resource availability can alter diversity-scaling relationships by changing the size of species pools and thus introduce differences in the coverage of sampling between treatments, due to larger and fewer individuals per area sampled ${ }^{28}$, and contribute to the loss of rarer species. We calculated species diversity as the effective species number, which estimates the probability of interspecific-encounter if all species are equally abundant (ESN $\left.\mathrm{PIE}_{\mathrm{PIE}}\right)$. $\mathrm{ESN}_{\mathrm{PIE}}$ has been shown to be less sensitive to scaling issues than other metrics ${ }^{22}$, and is representative of the maximum slope of the species-area accumulation function. We used ESN PIE because NutNet sites vary in their species pools and therefore their species accumulation curves will differ, which creates a challenge to compare species diversity when sampled at a fixed area ${ }^{22}$. ESN PIE has been shown to be relatively insensitive to such sampling area issues because it essentially measures the maximum change in species number as a function of sampling area (that is, the slope at the $x$ intercept of the species accumulation curve). Because the resource dimension hypothesis and underlying resource ratio theory assume that species trade-off for different limiting factors, predictions for diversity change describe changes in competitive dominance; ESN $_{\text {PIE }}$ captures these predicted changes in dominance better than simple measurements of local species extinction (that is, richness loss). We used the aggregate number of species observed at a site as an estimate of the asymptote of the species accumulation function, and of the regional species pool. We also used simply the number of species (that is, richness) and found similar results as those using ESN $N_{P I E}$ (Extended Data Table 1).

We measured species diversity annually by estimating the $\%$ cover of each plant species within a $1 \mathrm{~m} \times 1 \mathrm{~m}$ fixed location in each plot; the total cover typically summed to greater than $100 \%$ due to multiple canopy layers. We quantified species diversity as the probability of interspecific encounter (PIE), or effective species number (ESN PIE), assuming species relative abundances are equal:

$$
E S N_{P I E}=\frac{1}{\sum_{i}^{s} p_{i}^{2}}
$$

where $\mathrm{p}_{i}$ is the proportion of species $i$ in a community of size $s$; $E S N_{\mathrm{PIE}}$ is derived from the inverse of Simpson's diversity index ${ }^{22}$.

We measured aboveground live biomass by clipping two $1 \mathrm{~m} \times 10 \mathrm{~cm}$ strips of vegetation in each plot, sorting the sampled tissue to live (current year's production) and dead (previous years' production) fractions, drying at $60^{\circ} \mathrm{C}$ for $48 \mathrm{~h}$ and weighing. At most sites, photosynthetically active radiation PAR was measured above the plant canopy and at the ground surface and the proportion of transmitted light calculated.

We categorised plant communities at sites as multiple-resource limited if biomass responded positively to fertilisation with combinations of different nutrients. Specifically, we designated sites as 'multiple-resource limited' if biomass increased with the independent addition of different resources or if biomass responded synergistically to two or more added resources (that is, the response to one nutrient was dependent on the level of another and their combined effect was super-additive $)^{11}$. Sites that showed no response or negative biomass response or responded positively to only one resource we categorised as not multiple-resource limited. Thirty-four of the 45 sites showed increased biomass in response to multiple added resources; eight did not respond positively to resource addition, and three responded positively to a single resource (that is, single resource limited ${ }^{11}$ ). Statistical analysis. All analyses used $\mathrm{R}$ version 3.2.2. We used linear mixed-effects models ( $\mathrm{R}$ package lme) to test the interaction of number of added resources and the number of treatment years, on diversity $\left(\mathrm{ESN}_{\mathrm{PIE}}\right)$ and richness. Site and block were modelled as nested random effects. We included in the model an autocorrelation structure, a first-order autoregressive model $(\mathrm{AR}(1))$, where observations are expected to be correlated from one year to the next, and found a substantial improvement in model fit when we compared this model to a model with no autocorrelation structure (lower AIC $=\Delta 608$ and likelihood ratio tests, L.Ratio $=610$ $P<0.0001)^{29}$. Treatment effects increased in magnitude with time (significantly negative interaction between number of added resources and year; Supplementary Table 2). To allow standardized comparison of sites that differed in the year they were established and in duration of nutrient addition, we used two approaches to quantify the changes in species diversity. First, we calculated the annual rate of change of our response variables to standardise site responses. Second, for analyses that required an effect size, calculated as the log ratio of the treatment response divided by the control, we used the most recent year of treatment data, which ranged from 3 to 8 years of annual nutrient application duration (Supplementary Table 1). Log ratio effect size estimates would not have been possible using the rate of change estimates, which can take zero or negative values. Log ratio effect sizes tend to be normally distributed, centre zero effects (control levels) at zero log ratios, and scale responses to make proportional effects directly comparable between sites $^{30}$.

We used linear mixed-effects models (R package lme) to test the effects of number of treatment years, site richness, log live biomass, log dead biomass, PAR, total species cover, and the number of added resources on diversity $\left(\mathrm{ESN}_{\text {pie }}\right)$, with plot nested in block nested in site as random effects. Models using dead biomass and PAR used the subset of 32 sites for which we had data for these variables. We calculated mean values at each site for the annual rate of diversity loss and diversity effect size, and tested for linear relationships between these variables and the number of added resources using regression with site as a block term. We used step-wise linear regression and AIC criteria to test for relationships of loss of diversity (from addition of three resources) with latitude, longitude, and environmental covariates of mean annual precipitation, and soil $\mathrm{N}, \mathrm{P}, \mathrm{K}, \mathrm{pH}$, percentage clay, and percentage sand. Plant community composition changes were quantified using Bray-Curtis multivariate distances ( $\mathrm{R}$ package vegan).

27. Crawley, M. J. \& Harral, J. E. Scale dependence in plant biodiversity. Science $291,864-868$ (2001).

28. Oksanen, J. Is the humped relationship between species richness and biomass an artefact due to plot size? J. Ecol. 84, 293-295 (1996).

29. Pinheiro, J. \& Bates, D. Mixed-effects models in S and S-PLUS (Springer, 2006).

30. Hedges, L. V., Gurevitch, J. \& Curtis, P. S. The meta-analysis of response ratios in experimental ecology. Ecology 80, 1150-1156 (1999). 


\section{RESEARCH LETTER}

Extended Data Table 1 | The effects of nutrient addition on diversity loss and richness loss increase with time

\begin{tabular}{|c|c|c|c|c|}
\hline $\mathrm{ESN}_{\text {pie }}$ & Num. DF & Den. DF & $\mathrm{F}$ & $\mathrm{P}$ \\
\hline intercept & 1 & 6555 & 370.3 & $<0.0001$ \\
\hline year & 1 & 6555 & 39.3 & $<0.0001$ \\
\hline nres & 1 & 1049 & 32.2 & $<0.0001$ \\
\hline year $\mathrm{x}$ nres & 1 & 6555 & 26.1 & $<0.0001$ \\
\hline Richness & Num. DF & value & SE & $P$ \\
\hline intercept & 1 & 6555 & 146.4 & $<0.0001$ \\
\hline year & 1 & 6555 & 209.1 & $<0.0001$ \\
\hline res & 1 & 1049 & 91.8 & $<0.0001$ \\
\hline year $\mathrm{x}$ nres & 1 & 6555 & 33.5 & $<0.0001$ \\
\hline
\end{tabular}

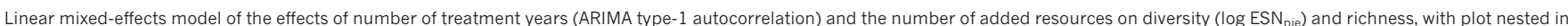
block, nested in year, nested in site, as random effects, using all 45 sites. There was a significant, negative interaction between the number of added resources ( $n$ res) and year of treatment (year). 
Extended Data Table 2 | The number of added resources predicts diversity loss after controlling for other variables

\begin{tabular}{lcccc}
\hline & Num. DF & Den. DF & F & P \\
intercept & 1 & 1029 & 329.9 & $<.0001$ \\
years of treatment & 1 & 42 & 12.1 & 21.5 \\
site richness & 1 & 42 & 17.4 & $<.0012$ \\
log live biomass & 1 & 1029 & 4.8 & $<0.0001$ \\
total cover & 1 & 1029 & 35.4 & $<.029$ \\
number of added resources & 1 & 1029 & $<0.0001$
\end{tabular}

Linear mixed-effects model of the effects of number of treatment years, site richness, log live biomass, total species cover, and the number of added resources on diversity (ESN pie), with plot nested in block nested in site as random effects, using all 45 sites and data from the maximum treatment year for each site. $\Delta$ AlC between model with number of added resources and model without was 33 , log-likelihood ratio 35.0, $P<0.0001$. 


\section{RESEARCH LETTER}

Extended Data Table 3 | The number of added resources is an important predictor even after controlling for other variables, for sites that had light and litter data

\begin{tabular}{lcccc}
\hline & Num. DF & Den. DF & F & $<.3$ \\
\hline intercept & 1 & 643 & 285.3 & 0.0001 \\
years of treatment & 1 & 29 & 14.1 & $<0.0001$ \\
site richness & 1 & 29 & 7.9 & 0.0052 \\
log live biomass & 1 & 643 & 4.5 & 0.034 \\
total cover & 1 & 643 & 0.34 & 0.56 \\
log dead biomass & 1 & 643 & 18.2 & $<0.0001$ \\
PAR & 1 & 643 & 15.6 & 0.0001 \\
number of added resources & 1 & 643 & & \\
\hline
\end{tabular}

Linear mixed-effects model of the effects of number of treatment years, site richness, log live biomass, log dead biomass, PAR, total species cover, and the number of added resources on diversity $\left(E S N_{\text {pie }}\right)$, with plot nested in block nested in site as random effects, using data from the maximum treatment year for each site, and the subset of 32 sites for which there was dead biomass and PAR data. $\triangle$ AIC between model with number of added resources and model without was 15, log-likelihood ratio $15.6, P<0.0001$. 
Extended Data Table 4 | Diversity loss due to addition of nutrients associated with soil properities

\begin{tabular}{lcccc}
\hline & DF & SS & F & P \\
\hline soil P & 1 & 0.16 & 1.72 & 0.20 \\
soil K & 1 & 0.018 & 0.20 & 0.66 \\
$\mathrm{pH}$ & 1 & 0.46 & 5.03 & 0.034 \\
\% sand & 1 & 0.73 & 8.05 & 0.0089 \\
residuals & 25 & 2.28 & & \\
\hline
\end{tabular}

Stepwise multiple regression (backward with AIC criteria for model comparisons) retained soil P, K, pH, and percentage sand as predictors of diversity loss from the addition of three resources, for the 30 sites with soil analysis data (excluding one site for extreme value of $\mathrm{P}$ ). The variables latitude, longitude, mean annual precipitation, and soil percentage $\mathrm{N}$ were not retained. Overall model is significant $\left(r^{2}=0.375, F_{4,25}=3.75, P=0.016\right)$. 